\title{
连分数与类数及其 他
}

\author{
陆 洪 文 \\ (中国科学技术大学数学系,合肥)
}

\section{·掊 要}

本文用连分数给出虚二次域的类数公式，见文中的定理 4. 结合 Hirzebruch 和 Zagier 的结果 (定理 5), 就完全了用连分数给出虚二次域的类数公式的结果. 另外 还讨论了一类虚二次域类数的可除性,即有

定理 6. 设 $l, q$ 均为正整数, 且 $q \geqslant 2$. 如 $0>\Delta=1-4 q^{l}$ 为无平方因子整 数, 则 $l$ 除尽虚二次域 $(Q, \sqrt{\Delta})$ 的类数.

文献 [6]中,用了代数几何方法证明了当 $l$ 为奇素数时的情形，本文只用初等方 法. 最后,文中给出了一个实二次域类数的初等公式.

\section{一、引言与结果}

设 $D>1$ 为一个无平方因子正整数, 而 $\Delta$ 是实二次域 $\boldsymbol{K}=\boldsymbol{Q}(\sqrt{D})$ 的判别式, 这儿 $\boldsymbol{Q}$ 是有理数域. $\boldsymbol{K}$ 中的每一个理想 $\mathscr{U}$ 在等价的意义下,可表为 ${ }^{[1]}$ :

$$
\mathfrak{A}=[A,( \pm B+\sqrt{\Delta}) / 2],
$$

这里 $A, B$ 均为有理整数,且有

$$
A \geqslant B \geqslant 0, B \equiv \Delta(\operatorname{mcd} 2), A \mid \frac{\Delta-B^{2}}{4},
$$

同时使有理整数

$$
C=\frac{\Delta-B^{2}}{4 A} \geqslant A
$$

及

$$
\text { g. c. d }(A, B, C)=1 \text {. }
$$

实二次无理数

$$
\alpha=\frac{ \pm B+\sqrt{\Delta}}{2 A}
$$

有简单连分数展开式

$$
\alpha=\left[a_{0}, \overline{a_{1}, \cdots, a_{k}}\right],
$$

其中 $\overline{a_{1}, \cdots, a_{k}}$ 为基本周期 (见文献 [3] (I)), 这里要指出, 如 


$$
\frac{B+\sqrt{\Delta}}{2 A}=\left[a_{0}, \overline{a_{1}, \cdots, a_{k}}\right]
$$

则有

$$
\frac{-B+\sqrt{\Delta}}{2 A}=\left[a_{k}-a_{0}, \overline{a_{k-1}, \cdots, a_{1}, a_{k}}\right]
$$

命

$$
\left[a_{m}, a_{m-1}, \cdots\right]=\frac{P_{m}+\sqrt{\Delta}}{2 Q_{m}} \quad(m \geqslant 0)
$$

为 $\alpha$ 的第 $m$ 个完全商, 则 $P_{m}, Q_{m}$ 均为非负整数. 我们有

定理 1. 设 $f(x)$ 是 $x$ 的任一个给定的函数, 则在上述记号下, 我们有

$$
\begin{aligned}
& \sum_{\substack{|b|<\sqrt{\Delta} \\
b \in \Delta(\bmod 2)}} \sum_{\substack{a \geq 1 \\
a \mid \frac{\left.\Delta \Delta b^{2}\right)}{4}}} f(a) \\
& =\sum_{(q)} \sum_{m=1}^{k} \sum_{t=1}^{a_{m}}\left(f\left(Q_{m}\right)+f\left(Q_{m+1}+t P_{m+1}-t^{2} Q_{m}\right)\right),
\end{aligned}
$$

其中 $\mathfrak{U}=\left[A, \frac{ \pm B+\sqrt{\Delta}}{2}\right]$ 跑过 $\boldsymbol{K}=\boldsymbol{Q}(\sqrt{\bar{D}})$ (广义) 理想类的完全代表元组.

在定理 1 中取 $f(x) \equiv 1$, 则有

定理 2. 在上述记号下, 我们有

$$
\sum_{\substack{|b|<\sqrt{\Delta} \\ t \equiv \Delta(\bmod 2)}} d\left(\frac{\Delta-b^{2}}{4}\right)=2 \sum_{\{\mathscr{N}\}} \sum_{m=1}^{k} a_{m},
$$

这里 $d(x)$ 是除数函数.

注 1. 定理 2 即文献 [2] 的 (4.10) 式.

在定理 1 中取 $f(x)=x$, 即有

定理 3. 在上述记号下,我们有

$$
605 \boldsymbol{K}(-1)=\sum_{\{y\}} \sum_{m=1}^{k} \sum_{t=1}^{a_{m}}\left(Q_{m}+Q_{m+1}+t P_{m+1}-t^{2} Q_{m}\right),
$$

这儿 $\zeta_{\boldsymbol{K}}$ 是 $\boldsymbol{K}$ 的 Dedekind $\boldsymbol{\zeta}$-函数

在定理 1 中取

$$
f(x)=\chi(x)=\left\{\begin{aligned}
1, & \text { 如 } x \equiv 1(\bmod 4), \\
-1, & \text { 如 } x \equiv 3(\bmod 4), \\
0, & \text { 如 } x \equiv 0(\bmod 2),
\end{aligned}\right.
$$

则有

定理 4. 在上述记号下,我们有

$$
\sum_{\{(1)\}} \sum_{m=1}^{k} \sum_{i=1}^{a_{m}}\left(\chi\left(Q_{m}\right)+\chi\left(Q_{m+1}+t P_{m+1}-t^{2} Q_{m}\right)\right)
$$




$$
=\left\{\begin{array}{cl}
h(-D), & \text { 如 } D \equiv 1(\bmod 4), \\
3 h(-D), & \text { 如 } D \equiv 2(\bmod 4), \\
6 h(-D), & \text { 如 } D=3(\bmod 8),
\end{array}\right.
$$

这里 $h(-D)$ 是虚二次域 $Q(\sqrt{-D})$ 的类数.

定理 与定理 5 完全给出了用连分数决定虚二次域类数的解答.

定理 5 (Hirzebruch 和 Zagier $\left.{ }^{[4]}\right)$. 对无平方因子正整数 $D \equiv 3(\bmod 4)$ ，设 $D>3$ ，则 在上述记号下，我们有

$$
3 h(-D)=\sum_{\{\mu\}} \chi_{1}(A) \sum_{m=1}^{k}(-1)^{m} a_{m}
$$

其中

$$
\chi_{1}(x)=\left\{\begin{array}{cl}
0, & \text { 如 } x \equiv 0(\bmod 4), \\
1, & \text { 如 } x \equiv 1(\bmod 4), \\
-1, & \text { 如 } x \equiv 3(\bmod 4), \\
\pm(-1)^{\left(D^{2}-1\right) / 8}, & \text { 如 } x \equiv \pm 2(\bmod 8) .
\end{array}\right.
$$

另外,对于一类虚二次域,我们可有

定理 6. 设 $l, q$ 为正整数, $q \geqslant 2$, 如 $0>\Delta=1-4 q^{l}$ 是无平方因子整数, 则 $l \mid h$, 其 中 $h$ 是虚二次域 $Q(\sqrt{\Delta})$ 的类数.

文献 [5] 证明了 $q, l$ 均为奇素数的情形; 文献 [6] 证明了 $l$ 为奇素数的情形,但它可去掉 $\Delta$ 为无平方因子的条件.

例. 取 $q=2, l=9$, 则 $\Delta=1-4 \times 2^{9}=-2047=-23 \times 89$, 而 $h(\boldsymbol{Q}(\sqrt{\Delta}))=18$, 所以 $9 \mid h$, 如所期待.

由定理 1 还可得到

定理 7. 设 $h, \varepsilon, \Delta$ 分别为实二次域 $Q(\sqrt{\Delta})$ 的类数, 基本单位与判别式, 则有

$$
\begin{aligned}
& \left.h=\frac{1}{2 \log \varepsilon} \sum_{\substack{1 \leqslant n<\sqrt{\Delta} \\
n \equiv \Delta(\bmod \Delta)}} \sum_{\frac{\sqrt{\Delta}-n}{2}<m<\frac{\sqrt{\Delta}+n}{2}} 1\right) \log \frac{\sqrt{\Delta}+n}{\sqrt{\Delta}-n} . \\
& m \mid \frac{\Delta-n^{2}}{4}
\end{aligned}
$$

定理 7 相应于虚二次域中用初等方式给出类数的公式，即对以 $-\Delta<0$ 为基本判别式之 虚二次域 $Q(\sqrt{-\Delta})$, 其类数为适合于

$$
b^{2}-4 a c=-\Delta,\left\{\begin{array}{l}
-a<b \leqslant a<c ， \\
\text { 或 } \iota \leqslant b \leqslant a=c
\end{array}\right.
$$

的整数组 $a, b, c$ 的组数, 可见文献 [1] p. 337. 定理 3.

\section{二、定理 1 的证明}

我们考虑实二次域 $\boldsymbol{Q}(\sqrt{D})$ 中的所谓的“约化” 的实二次无理数. 熟知

$$
\omega=\frac{P+\sqrt{\Delta}}{2 Q}\left(P, Q \text { 有理整数, } Q \neq 0, P \equiv \Delta(\bmod 2), Q \mid \frac{P^{2}-\Delta}{4}\right)
$$


称为“约化”的, 是指

$$
\omega=\frac{P+\sqrt{\Delta}}{2 Q}>1>\omega^{\prime}=\frac{P-\sqrt{\Delta}}{2 Q}>0 .
$$

由文献 [2]即知, 满足条件

$$
\left[Q, \frac{P+\sqrt{\Delta}}{2}\right] \sim \mathfrak{U}^{*}=[A,(\mp B+\sqrt{\Delta}) / 2]=A \mathfrak{A}^{-\mathfrak{1}}
$$

的 “约化”的实二次无理数 $\omega=\frac{P+V^{\prime} \Delta}{2 Q}$ 的个数为:

$$
I_{\mathfrak{U}}=\sum_{m=1}^{k} a_{m},
$$

上逄的 婊示两个留想的等价. 年一方面, 上述“约化”的实二次无理数的总数是

$$
\begin{aligned}
& l_{D}=\sum_{\frac{P+\sqrt{\Delta}}{2 Q}>1>\frac{P-\sqrt{\Delta}}{2 Q}>0} 1=\sum_{\mid P-2 Q^{\prime}<\sqrt{\Delta}, P>\sqrt{\Delta}} 1
\end{aligned}
$$

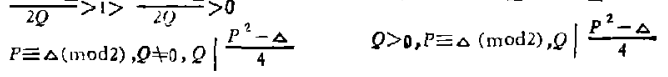

$$
\begin{aligned}
& =\sum_{\substack{|b|<\sqrt{\Delta}, l \equiv \Delta(\bmod 2), a>1 \\
a \mid \frac{\Delta-b^{2}}{4}, \frac{\sqrt{\Delta}-b}{2 \cdot a}<1}} 1=\sum_{\substack{a \geqslant 1, u \\
\mid \frac{\Delta}{4}, \frac{\sqrt{\Delta}}{2 a}<1}} 1 \\
& +\sum_{1 \leqslant b<\sqrt{\Delta,} ! \equiv \Delta(\bmod 2), \alpha \geqslant 1} 1+\sum_{1 \leqslant b<\sqrt{\Delta}, ! \equiv \Delta(\bmod 2), a>1} 1 \text {, } \\
& a \mid \frac{\Delta-b^{2}}{4}, \frac{\sqrt{\Delta}-b}{2 a}<1 \quad \text { a } \frac{\Delta-b^{2}}{4}, \frac{\sqrt{\Delta}+b}{2 a}<1
\end{aligned}
$$

以上第一个和当 $4 \nmid \Delta$ 时是 0 , 当 $4 ! \Delta$ 时, 是

$$
\begin{aligned}
& \sum_{\sqrt{-}} 1=\sum_{\sqrt{-}} 1=\frac{1}{2} d\left(\frac{\Delta}{4}\right) . \\
& a 21, a\left|\frac{\Delta}{4}, \frac{\sqrt{-}}{2, a}<1 \quad a \geqslant 1, a\right| \frac{\Delta}{4}, \frac{\sqrt{\Delta}}{2, a}>1
\end{aligned}
$$

又易见

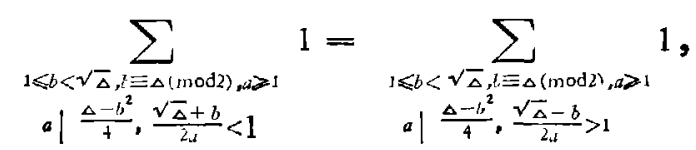

故得

$$
I_{D}=\frac{1}{2} \sum_{\substack{|b|<\sqrt{\Delta}, l \equiv \Delta(\bmod 2) \\ a \geq 1, a \mid}} 1
$$

由此即得定理 2(上述证明实质上属于 Hirzebruch 与 Zagier) ${ }^{[2]}$.

由定理 2 的证明可知, 满足

$$
\left[Q, \frac{P+\sqrt{\Delta}}{2}\right] \sim \mathfrak{A}^{*}=\left[A, \frac{\mp B+\sqrt{\Delta}}{2}\right]=A \mathfrak{X}^{-\mathfrak{l}}
$$


的所有 “约化” 的实二次无理数 $\omega=\frac{p+\sqrt{\Delta}}{2 Q}$ 形如下述:

$$
\begin{aligned}
& Q=Q_{m+1}+t P_{m+1}-t^{2} Q_{m}, \\
& P=\left(2 Q_{m+1}-P_{m+1}\right)+2 t\left(F_{m+1}+Q_{m}\right)-2 t^{2} Q_{m}, \\
& 1 \leqslant t \leqslant a_{m}, \quad 1 \leqslant m \leqslant k .
\end{aligned}
$$

由此易知上述所列的 $\frac{P+\sqrt{\Delta}}{2 Q}$ 是“约化”的, 且任意这样两个数相等的充要条件是 $\imath$ 与 $m$ 均相 等,而它们的总数为 $I_{2}$.

由此, 注意到, 对上述的 $\frac{P+\sqrt{\Delta}}{2 Q}$ 有

$$
\frac{\Delta-(P-2 Q)^{2}}{4 Q}=Q_{m} \text {. }
$$

因此类似于定理 2 的证明,可以知道 $\mathfrak{U}=\left[A, \frac{\mp B+\sqrt{\Delta}}{2}\right]$ 对定理 1 中等式左端的所提供的 值正好是

$$
\sum_{m=1}^{k} \sum_{i=1}^{a_{m}}\left(f\left(Q_{m}\right)+f\left(Q_{m+1}+t P_{m+1}-t^{\prime} Q_{m}\right)\right)
$$

这样即得定理 1 .

注 1. 如考虑㹨义理想类, 即可得定理 5, 可见文献 [4]

注 2. 定理 4 的证明用二平方和与三平方和定理即可得到.

\section{三、定理 6 的证明}

我们先证明下列简单的

引理 1. 设 $D$ 为无平方因子整数, $D$ 可正可负. 再设

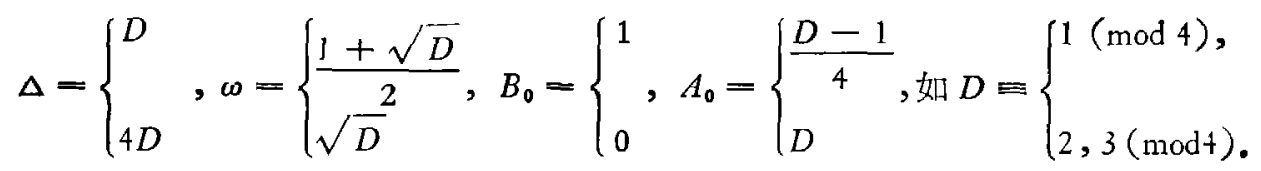

设二次域 $\boldsymbol{Q}(\sqrt{\Delta})$ 中的二个理想

$$
\mathfrak{A}=[a, b+\omega] \text { 与 } \mathfrak{B}=\left[a_{1}, b_{1}+\omega\right]
$$

满足条件

$$
\Delta=\left(2 b+B_{0}\right)^{2}+4 a a^{\prime}=\left(2 b_{1}+B_{0}\right)^{2}+4 a_{1} a_{1}^{\prime},
$$

其中 $a, b, a^{\prime}, a_{1}, b_{1}, a_{1}^{\prime}$ 均为有理整数. 那么我们有

这里

$$
\mathfrak{U} \mathfrak{B}=c\left[x x_{1}, v+\omega\right],
$$

$$
\begin{aligned}
& c=\text { g. c. d. }\left(a, a_{1}, b+b_{1}+B_{0}\right), \\
& a=c x, a_{1}=c x_{1}, b+b_{1}+B_{0}=c y, \\
& x z+x_{1} z_{1}+y u=1, \\
& v=b+\left(b_{1}-b\right) x z+a^{\prime} x u=b_{1}+\left(b-b_{1}\right) x_{1} z_{1}+a_{1}^{\prime} x_{1} u,
\end{aligned}
$$


其中 $c, x, y, z, x_{1}, z_{1}, u, v$ 均为有理整数.

证. 由 $\omega^{2}=B_{0} \omega+A_{0}$, 即有

$$
\mathfrak{2} \mathfrak{B}=\left[a a_{1}, a b_{1}+a \omega, a_{1} b+a_{1} \omega, b b_{1}+A_{0}+\left(b+b_{1}+B_{0}\right) \omega\right] .
$$

而今有

$$
B_{0}+4 A_{0}=\Delta=\left(2 b+B_{0}\right)^{2}+4 a a^{\prime}=\left(2 b_{1}+B_{0}\right)^{2}+4 a_{1} a_{1}^{\prime},
$$

故得

$$
\begin{aligned}
& A_{0}=b^{2}+b B_{0}+a a^{\prime}=b_{1}^{2}+b_{1} B_{0}+a_{1} a_{1}^{\prime}, \\
& 0=\left(b-b_{1}\right)\left(b+b_{1}+B_{0}\right)+a a^{\prime}-a_{1} a_{1}^{\prime}, \\
& \left(b-b_{1}\right) y=a_{1}^{\prime} x_{1}-a^{\prime} x, b y+a^{\prime} x=b_{1} y+a_{1}^{\prime} x_{1}, \\
& A_{0}+b b_{1}=\frac{1}{2}\left(\left(b+b_{1}\right)\left(b+b_{1}+B_{0}\right)+a a^{\prime}+a_{1} a_{1}^{\prime}\right) \\
& =\frac{c}{2}\left(\left(b+b_{1}\right) y+a^{\prime} x+a_{1}^{\prime} x_{1}\right) \\
& =c\left(b y+a^{\prime} x\right)=c\left(b_{1} y+a_{1}^{\prime} x_{1}\right),
\end{aligned}
$$

从而

$$
\begin{aligned}
\mathfrak{A B}= & c\left[c x x_{1}, b_{1} x+x \omega, b x_{1}+x_{1} \omega, b y+a^{\prime} x+y \omega\right] \\
= & c\left[c x x_{1}, b_{1} x+x \omega, b x_{1}+x_{1} \omega, b y+a^{\prime} x+y \omega, b_{1} y+a_{1}^{\prime} x_{1}+y \omega,\right. \\
& \left.b_{1} x z+b_{1} x_{1} z_{1}+\left(b y+a^{\prime} x\right) u+\omega, b_{1} x z+b x_{1} z_{1}+\left(b_{1} y+a_{1}^{\prime} x_{1}\right) u+\omega\right] \\
= & c\left[c x x_{1}, b_{1} x+x \omega, b x_{1}+x_{1} \omega, b y+a^{\prime} x+y \omega, b_{1} y+a_{1}^{\prime} x_{1}+y \omega,\right. \\
& \left.\left(b_{1}-b\right) x z+b+a^{\prime} x u+\omega,\left(b-b_{1}\right) x_{1} z_{1}+b_{1}+a_{1}^{\prime} x_{1} u+\omega\right] \\
= & c\left[c x x_{1},\left(b_{1}-b\right) x_{1} z_{1} x-a_{1}^{\prime} x_{1} u x,\left(b-b_{1}\right) x z x_{1}-a^{\prime} x u x_{1},\right. \\
& a^{\prime} x+\left(b-b_{1}\right) x z y-a^{\prime} x u y, a_{1}^{\prime} x_{1}+\left(b_{1}-b\right) x_{1} z_{1} y-a_{1}^{\prime} x_{1} u y, \\
& \left.\left(b_{1}-b\right) x z+b+a^{\prime} x u+w,\left(b-b_{1}\right) x_{1} z_{1}+b_{1}+a_{1}^{\prime} x_{1} u+\omega\right],
\end{aligned}
$$

但是

$$
\begin{aligned}
& a^{\prime} x+\left(b-b_{1}\right) x z y-d x u y=x\left(a^{\prime}+\left(b-b_{1}\right) y z-a^{\prime} u y\right) \\
& =x\left(a^{\prime}+a_{1}^{\prime} x_{1} z-a^{\prime} x z-a^{\prime} u y\right)=x\left(a_{1}^{\prime} x_{1} z+a^{\prime}(1-x z-u y)\right) \\
& =x x_{1}\left(a_{1}^{\prime} z+a^{\prime} z_{1}\right)=a_{1}^{\prime} x_{1}+\left(b_{1}-b\right) x_{1} z_{1} y-a_{1}^{\prime} x_{1} u y,
\end{aligned}
$$

于是我们得到

$$
\mathfrak{A} \mathfrak{B}=c\left[x x_{1} v_{1}, v+\omega\right]
$$

其中

$$
v_{1}=\mathrm{g} . c . \mathrm{d}\left(c,\left(b_{1}-b\right) z_{1}-a_{1}^{\prime} u,\left(b-b_{1}\right) z-a^{\prime} u, a_{1}^{\prime} z+a_{z_{1}}^{\prime}\right) .
$$

由假设知 $a, b+\omega$ 是 $\mathfrak{A}$ 的整基, $a_{1}, b_{1}+\omega$ 是 $\mathfrak{B}$ 的整基, 并由证明过程可见 $c x x_{1} v_{1}, c(v+\omega)$ 是 $\mathfrak{A B}$ 的整基, 所以由

$$
a a_{1}=N(\mathfrak{A}) N(\mathfrak{B})=N(\mathfrak{H B})=c^{2} N\left(\left[x x_{1} v_{1}, \nu+\omega\right]\right)=c^{2} x x_{1} v_{1},
$$

及

$$
a a_{1}=c^{2} x x_{1},
$$

即知 $\nu_{1}=1$, 故得引理 1 .

定理 6 的证明. 


\section{第一步来证明}

断言 1. 如命 $\boldsymbol{Q}(\sqrt{\Delta})$ 中的理想

$$
\mathfrak{A}=[q,(1)],
$$

其中 $\Delta=1-4 q^{l}$, 则有

$$
2 l^{n}=\left[q^{n}, \omega\right], \text { 对 } 1 \leqslant n \leqslant l .
$$

证. $n=1$ 时显然成立, 以下利用归纳. 设对 $n=m \leqslant l-1$, 断言成立, 即有

$$
\mathfrak{2}^{m}=\left[q^{m}, \omega\right] \text {. }
$$

取上述引理中的 $\mathfrak{B}=\mathfrak{A}^{m}=\left[q^{m}, \omega\right]$, 则引理中的相应有理整数如下所示:

$$
\begin{aligned}
& c=\text { g. c. d }\left(q, q^{m}, 1\right)=1, a^{\prime}=-q^{l-1}, \\
& x=q, x_{1}=q^{m}, y=1, z=1, z_{1}=0, u=1-q, \\
& v=-q^{l-1} \cdot q \cdot(1-q)=q^{l}(q-1),
\end{aligned}
$$

故由引理得

$$
\mathfrak{U}^{n+1}=\left[q^{m+1}, q^{l}(q-1)+\omega\right],
$$

但 $m+1 \leqslant l$, 故得

$$
\mathfrak{U}^{m+1}=\left[q^{m+1},(n],\right.
$$

由归纳法原理知断言 1 得证。

断言 2. 如断言 1 所设, 则有

对 $1 \leqslant n \leqslant l, \mathfrak{X}^{n} \sim[1]$ 当且仅当 $n=l$.

证. 在上述有关的说明下,即得

$$
\mathfrak{U}^{l}=\left[q^{l}, \omega\right] \sim[1] .
$$

如某个 $n, l \leqslant n \leqslant l-1$, 使

$$
\mathfrak{A}^{n}=\left[q^{n}, \omega\right] \sim[1],
$$

则必存在有理整数 $r, s, t, u$, 使

$$
\begin{gathered}
r u-t s=1, \\
q^{n}=r^{2}-r t+q^{l} t^{2} .
\end{gathered}
$$

故

$$
4 q^{n}=(2 r-t)^{2}+\left(4 q^{l}-1\right) t^{2} .
$$

由 $q \geqslant 2,1 \leqslant n \leqslant l-1$, 即知 $t=0$, 故 $r=u= \pm 1$, 从而

$$
q^{n}=1 \text {, }
$$

此不可能,断言 2 得证.

由断言 2 即知, $\mathfrak{A}$ 在 $\boldsymbol{Q}(\sqrt{\Delta})$ 理想类群中的阶为 $l$, 故 $l$ 除尽所述 理 想类群 的阶, 即 $\mathbf{Q}(\sqrt{\Delta})$ 的类数 $h$, 即有 $l \mid h$.

定理 6 证完.

\section{四、定理 7 的证明}

我们在第二节的“约化”的实二次无理数 $\omega=\frac{P+\sqrt{\Delta}}{2 Q}$ 中取下列的, 即满足条件 


$$
\omega=\frac{P+\sqrt{\Delta}}{2 Q}>2,0<\omega^{\prime}=\frac{P-\sqrt{\Delta}}{2 Q}<1 .
$$

令

$$
m=Q, n=P-2 Q,
$$

那么可知上述 $\omega$ 与满足

$$
\begin{gathered}
0<\frac{\sqrt{\Delta}-n}{2 m}<1<\frac{\sqrt{\Delta}+n}{2 m}(m, n \text { 有理整数, } \\
\left.n \geqslant 1, n \equiv \Delta(\bmod 2), 1 \leqslant m \mid \frac{\Delta-n^{2}}{4}\right)
\end{gathered}
$$

的 $\frac{n+\sqrt{\Delta}}{2 m}-$ 一对应: 再由定理 1 证明中的 $P, Q$ 所具有形式知, 如

$$
\left[Q, \frac{P+\sqrt{\Delta}}{2}\right] \sim\left[A, \frac{\mp B+\sqrt{\Delta}}{2}\right]
$$

则

$$
P-2 Q=2 t Q_{l}-P_{l+1},(P-2 Q)^{2}+4 Q Q_{l}=\Delta, 1 \leqslant t \leqslant a, 1 \leqslant l \leqslant k .
$$

从而条件 $\omega>2,0<\omega^{\prime}<1, \omega=\frac{P+\sqrt{\Delta}}{2 Q}$ 表明

$$
2<\frac{P+\sqrt{\Delta}}{2 Q}=1+\frac{P-2 Q+\sqrt{\Delta}}{2 Q}=1+\frac{2 Q_{m}}{\sqrt{\Delta}-(P-2 Q)},
$$

故

$$
\begin{aligned}
& \frac{\sqrt{\Delta}-2 \iota Q_{l}+P_{l+1}}{2 Q_{l}}<1, \\
& a_{l}-t+\frac{\sqrt{\Delta}-P_{l}}{2 Q_{l}}<1 .
\end{aligned}
$$

由 $0<\frac{\sqrt{\Delta}-P_{l}}{2 Q_{l}}<1$, 即知 $a_{l}-t=0$, 故 $t=a_{l}$ 于是

$$
n=P-2 Q=P_{l}, \quad m=Q=Q_{l-1} .
$$

所以满足 (2) 式的 $\frac{\sqrt{\Delta}+n}{2 m}$, 如 $\left[m, \frac{n+\sqrt{\Delta}}{2}\right] \sim\left[A, \frac{\mp B+\sqrt{\Delta}}{2}\right]$, 则是且仅是

再由文献 [7]引理 6 可知,

$$
\frac{\sqrt{\Delta}+P_{l}}{2 Q_{l-1}}, 1 \leqslant l \leqslant k \text {. }
$$

$$
\varepsilon=p_{k-1}+q_{k-1} \frac{\sqrt{\Delta}-B}{2 A}
$$

这里 $\frac{p_{l}}{q l}=\left[a_{0}, a_{1}, \cdots, a_{l}\right]$ 是 


$$
\frac{B+\sqrt{\Delta}}{2 A}=\left[a_{0}, \overline{a_{1}, \cdots, a_{k}}\right]
$$

的第 $l$ 个渐近分数. 用归纳法, 容易证明

$$
\theta_{0} \theta_{1} \cdots \theta_{l}=p_{l-1} \theta_{l}+p_{l-2},
$$

这里

$$
\theta_{l}=\left[a_{l}, a_{l+1}, \cdots\right](l \geqslant 0)
$$

是 $\frac{E+\sqrt{\Delta}}{2 A}$ 的第 $l$ 个完全商 $\left(l=1\right.$ 时为显然, 归纳步聚中用 $\theta_{l}=a_{l}+\frac{1}{\theta_{l+1}}$ 及 $p_{l}=a_{l} p_{l-1}+$ $\left.p_{1-2}\right)$. 从而由 $\varepsilon$ 的表示式可得

$$
\begin{aligned}
& \theta_{0} \theta_{1} \theta_{2} \cdots \theta_{k}=p_{k-1} \theta_{k}+p_{k-2}=p_{k-1}\left(a_{k}-a_{0}+\theta_{0}\right)+p_{k-1} \\
& =\varepsilon \theta_{0}+p_{k}-a_{0} p_{k-1}-q_{k-1} \frac{\sqrt{\Delta}-B}{2 A} \frac{\sqrt{\Delta}+B}{2 A} \\
& =\varepsilon \theta_{0}+p_{k}-a_{0} p_{k-1}-q_{k-1} \frac{c}{A} .
\end{aligned}
$$

再由作者另一文 ${ }^{1)}$ 引理 6 证明的过程,即知有

$$
p_{k}-a_{0} p_{k-1}-q_{k-1} \frac{c}{A}=0,
$$

妓得

$$
\theta_{0} \theta_{1} \theta_{2} \cdots \theta_{k}=\varepsilon \theta_{0}
$$

从而

$$
\varepsilon=\prod_{l=1}^{k} \theta_{l}=\prod_{l=1}^{k} \frac{p_{l}+\sqrt{\Delta}}{2 Q_{l}},
$$

易见这对 $\frac{-B+\sqrt{\Delta}}{2 A}$ 也成立. 于是得到(注甞 $Q_{0}=Q_{k}$ )

$$
\varepsilon=\prod_{\substack{0<\frac{\sqrt{\Delta}-12}{2 m}<1<\frac{\sqrt{\Delta}+n}{\bar{m} m} \\ 1 \leqslant n \equiv \Delta(\bmod 2) \\ s \leqslant\left. m\right|^{\Delta-\frac{n^{2}}{4}}}} \frac{n+\sqrt{\prime \Delta}}{2 m},
$$

这里 $\mathfrak{x}=\left[A, \frac{\mp B+\sqrt{\Delta}}{2}\right]$. 所以

$$
h \log \varepsilon=\sum_{\substack{1 \leqslant n<\sqrt{\Delta} \\ n \equiv \Delta(\bmod 2)}} \sum_{\substack{\sqrt{\Delta}-n \\ 2} \frac{m<\frac{\sqrt{\Delta}+n}{2}}{\left.m\right|^{\frac{\Delta-n^{2}}{4}}}} \log \frac{n+\sqrt{\Delta}}{2 m}
$$

1)“实二次元理数连少数展开式的周期”一文.即将尖表. 


$$
=\frac{1}{2} \sum_{\substack{1 \leqslant n<\sqrt{\Delta} \\ n \equiv \Delta\left(\bmod _{2}\right)}}\left(\sum_{\substack{\sqrt{\Delta}-n \\ 2 \\ m \mid \frac{\Delta-n^{2}}{4}}} 1\right) \log \frac{\sqrt{\Delta}+n}{\sqrt{\Delta}-n},
$$

上面用到 $\Delta-n^{2}=4 m^{\prime}$ ，再作适当变换即得结果. 这就证明了定理 7 .

\section{考文献}

[1] 华罗庚,数论导引,科学出版社, 1957 .

[2] Hirzebruch, F. \& Zagier, D.. Classification of Hilbert Modular Surface, A Colletion of Papers Dedicated to K. Kodaira. 1977.

[3] 陆共文, (i) Scientia Sinica, Special issul (II), 1979, 118-130; (ii) Scientia Sinica, 24(1981), $1352-1357$.

[4] Zagier, D., Asterisque, 24-25(1975), 81-97.

[5] Cowles, M. J., J. Number Theory, 12 (1980), 113-115.

[6] Gross, B. \& Rohrlich, D., Invent. Math., 44(1978), 201-204.

[ 7 ] 陆猉文,数学学根(即将发表). 